\title{
FM-CW millimeter wave demonstrator system as a sensor of the sea surface wind vector
}

\author{
Alexey Nekrasov, ${ }^{1 \text { a) }}$ Jacco J.M. de Wit, ${ }^{2}$ and Peter Hoogeboom ${ }^{2}$ \\ ${ }^{1}$ Taganrog State University of Radio Engineering \\ 1 Krepostnoy 34, k. 249, 347922 Taganrog, Rostov Region, Russia \\ ${ }^{2}$ IRCTR, Delft University of Technology \\ Mekelweg 4, 2628 CD Delft, The Netherlands \\ a) alexei-nekrassov@mail.ru
}

Abstract: A small, low-cost FM-CW demonstrator system has been designed to investigate the feasibility of the application of FM-CW SAR in the field of airborne earth observation. A Ka-band backscatter model function and an algorithm for measurement of the wind vector over the water surface by this demonstrator system in the scatterometer mode have been developed. The principle considered, backscatter model and measuring algorithm proposed can be used for creating an airborne radar system for operational measurement of the sea roughness characteristics and for safe landing of an amphibious airplane on water, in particular under search and rescue missions in coastal areas. Keywords: FM-CW radar, millimeter wave system, backscatter model, algorithm

Classification: Microwave and millimeter wave devices, circuits, and systems

\section{References}

[1] R. C. Beal, Directional Ocean Wave Spectra: Measuring, Predicting, and Applying, The Johns Hopkins University Press, Baltimore and London, p. 218, 1989.

[2] F. M. Henderson and A. J. Lewis, Manual of Remote Sensing, vol. 2. Principles and Applications of Imagining Radar, 3rd ed., John Wiley \& Sons, New York, p. 866, 1998.

[3] D. Hauser, G. Caudal, G. -J. Rijckenberg, D. Vidal-Madjar, G. Laurent, and P. Lancelin, "RESSAC: A New Airborne FM/CW Radar Ocean Wave Spectrometer," IEEE Trans. Geosci. Remote Sensing, vol. 30, no. 5, pp. 981-995, Sept. 1992.

[4] J. J. M. de Wit and P. Hoogeboom, "High Resolution FM-CW SAR Performance Analysis," Proc. IGARSS 2003, Toulouse, France, vol. 7, pp. 4317-4319, 21-25 July 2003.

[5] H. Masuko, K. Okamoto, M. Shimada, and S. Niwa, "Measurement of Microwave Backscattering Signatures of the Ocean Surface Using X Band and 
Ka Band Airborne Scatterometers," J. Geophys. Res., vol. 91, no. C11, pp. 13065-13083, Nov. 1986.

[6] J. -P. Giovanangeli, L. F. Bliven, and O. L. Calve, "A Wind-Wave Tank Study of the Azimuthal Response of a Ka-Band Scatterometer," IEEE Trans. Geosci. Remote Sensing, vol. 29, no. 1, pp. 143-148, Jan. 1991.

[7] P. H. Hildebrand, "Estimation of Sea-Surface Wind Using Backscatter Cross-Section Measurements from Airborne Research Weather Radar," IEEE Trans. Geosci. Remote Sensing, vol. 32, no. 1, pp. 110-117, Jan. 1994.

\section{Introduction}

In the field of airborne earth observation, there is growing interest in small, cost effective radar systems. Such radar systems should consume little power and be small enough to be mounted on light, possibly even unmanned, aircraft. Frequency modulated continuous wave (FM-CW) radar systems are generally compact, relatively cheap, and they consume little power. Consequently, FM-CW radar technology seems to be of interest to civil airborne earth observation, particularly in combination with high resolution synthetic aperture radar (SAR) techniques.

During last decades, SAR techniques have successfully demonstrated their potential for microwave remote sensing of the sea surface. SAR provides an image of the roughness distribution on the water surface. Retrieval of seawave parameters and near-surface wind from SAR images provides a useful complement to support traditional measurements [1,2].

At the same time, the validity of the principle to derive the spectra of sea waves and other water surface parameters from real-aperture airborne radars (without the constraints of SAR techniques) has been demonstrated by a Ku-band noncoherent pulse-compression radar ROWS (Radar Ocean Wave Spectrometer) developed in the USA [1], and by a C-band FM-CW system RESSAC (Radar pour l'Etude du Spectre des Surfaces par Analyse Circulaire) developed in France [3].

Recently, a small, low-cost Ka-band FM-CW SAR demonstrator system has been developed in the Netherlands in order to study the feasibility of FM-CW SAR for airborne earth observation applications [4]. Earlier, a Kaband backscatter from the water surface has been studied using airborne [5] and wind-wave tank [6] scatterometer experiments.

In this paper, the IRCTR's airborne Ka-band FM-CW SAR demonstrator system, the principle of estimation of the wind vector over the sea surface using the system in a scatterometer mode, the proposed backscatter model and the measurement geometry are discussed.

\section{FM-CW demonstrator system}

In order to prove the feasibility of the concept of FM-CW SAR under operational circumstances, a small, low-cost, high-resolution demonstrator system 
has been developed. In addition, the demonstrator system should provide experimental data to validate the proposed measurement algorithm.

The Stemme S10 motorglider provides a relatively cheap test platform. For this motorglider standard under wing pods are available. These pods have a diameter of about $35 \mathrm{~cm}$ and a length of $80 \mathrm{~cm}$, excluding the aerodynamic fairings. The maximum payload of the pods is $50 \mathrm{~kg}$. The complete demonstrator system has been fitted in such a pod (Fig. 1a).

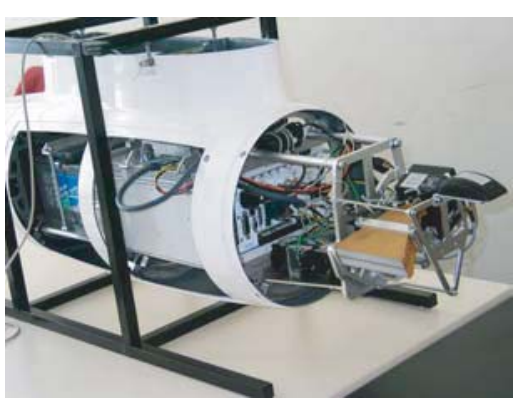

a)

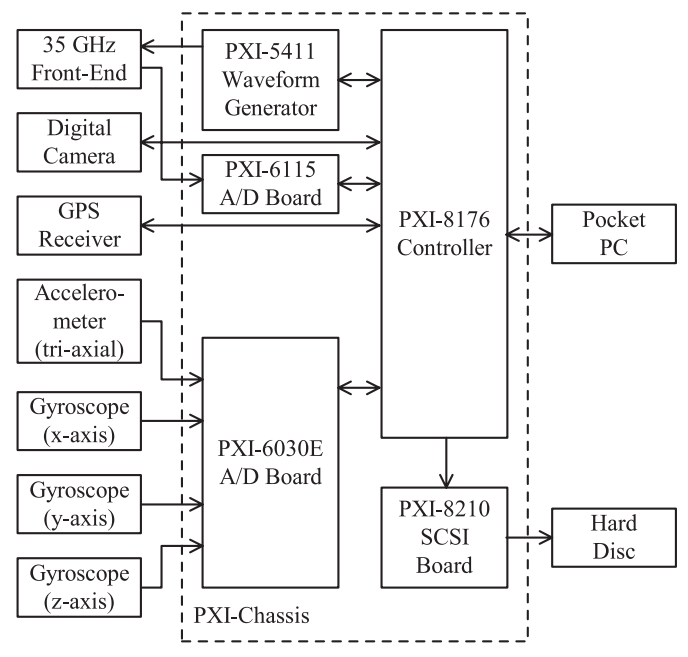

b)

Fig. 1. The FM-CW demonstrator system: a) the system installed in the standard pod; b) the block diagram.

Over land, the demonstrator operates in stripmap mode. The antenna axis is directed to the right side at a mounting incidence angle of $65^{\circ}$. The resolution is $1 \mathrm{~m}$ in range direction as well as in azimuth direction. Further specifications of the demonstrator system are summarized in Table I.

The core of the demonstrator system is a PXI-chassis manufactured by National Instruments Corporation. The chassis includes an embedded controller, two A/D boards, a wide ultra SCSI board, and an arbitrary waveform

Table I. Specifications of the FM-CW demonstrator system.

\begin{tabular}{|l|c|}
\hline Carrier Frequency & $35 \mathrm{GHz}$ \\
\hline Frequency Sweep & $500 \mathrm{MHz}$ \\
\hline Sweep Repetition Frequency & $1000 / 500 \mathrm{~Hz}$ \\
\hline Modulation & Sawtooth/Triangular \\
\hline Transmitted Power & $18 \mathrm{dBm}(0.063 \mathrm{~W})$ \\
\hline IF Band & 0 to $2.5 \mathrm{MHz}$ \\
\hline Antenna Type & Horn-Lens \\
\hline Antenna Gain & $24 \mathrm{~dB}$ \\
\hline Antenna Isolation & $52 \mathrm{~dB}$ \\
\hline Beamwidth Azimuth/Elevation & $6^{\circ} / 28^{\circ}$ \\
\hline Platform Velocity & $30 \mathrm{~m} / \mathrm{s}$ \\
\hline Altitude & $\leq 300 \mathrm{~m}$ \\
\hline Maximum Range & $730 \mathrm{~m}$ \\
\hline
\end{tabular}


generator. The control software of the chassis is written in LabVIEW. The block diagram of the FM-CW SAR demonstrator is presented in Fig. 1b.

The $35 \mathrm{GHz}$ front end is manufactured by Epsilon-Lambda Electronics. The frequency sweep and the modulation scheme are controlled by the PXI5411 waveform generator and they are both software selectable. The radar data are sampled by the PXI-6115 fast A/D board at $5 \mathrm{MHz}$. The IF-band is therefore limited to 0 to $2.5 \mathrm{MHz}$. The resulting continuous data rate is around 9.5 Mbytes/s.

The demonstrator system comprises a tri-axial accelerometer and three gyroscopes in order to be able to estimate the attitude and the position of the motorglider. The motion data are sampled by the PXI-6030E slow A/D board at $1 \mathrm{kHz}$. The motion sensors show however a considerable drift over time, a Global Positioning System (GPS) receiver has therefore been added. With the aid of the GPS readings the position estimate from the motion data can be updated. The GPS receiver outputs its position once every second through a RS232 connection.

A digital camera provides pictures of the area imaged by the radar. The camera is controlled by the embedded controller via a USB link.

During the flights, the demonstrator system can be controlled and monitored from the cockpit with a pocket PC through a standard network connection.

\section{Measuring algorithm}

Since the Ka-band normalized radar cross section (NRCS) model function was not available but is necessary for the water surface application of the FM-CW demonstrator system, we decided to use available NRCS model function for up-, down- and cross-wind directions from [5] to develop the azimuthal model function at least for the incidence angles of $30^{\circ}$ to $50^{\circ}$. It can be described in the widely used form [7]

$$
\sigma^{\circ}(U, \theta, \alpha)=A(U, \theta)+B(U, \theta) \cos \alpha+C(U, \theta) \cos (2 \alpha),
$$

where $A(U, \theta), B(U, \theta)$ and $C(U, \theta)$ are the Fourier terms that depend on sea surface wind speed $U$ and incidence angle $\theta, A(U, \theta)=a_{0}(\theta) U^{\gamma_{0}(\theta)}, B(U, \theta)=$ $a_{1}(\theta) U^{\gamma_{1}(\theta)}$, and $C(U, \theta)=a_{2}(\theta) U^{\gamma_{2}(\theta)} ; a_{0}(\theta), a_{1}(\theta), a_{2}(\theta), \gamma_{0}(\theta), \gamma_{1}(\theta)$ and $\gamma_{2}(\theta)$ are the coefficients dependent on the incidence angle. The following coefficients for the Ka-band NRCS model of form (1) for the incidence angles of $30^{\circ}$ to $50^{\circ}$ were computed

$$
\begin{gathered}
a_{0}(\theta)=0.006036-0.0002031 \theta+0.00000168 \theta^{2}, \\
a_{1}(\theta)=-0.007776+0.0004421 \theta-0.000005692 \theta^{2}, \\
a_{2}(\theta)=0.001129+0.00001335 \theta-0.0000006895 \theta^{2}, \\
\gamma_{0}(\theta)=4.902-0.198 \theta+0.00281 \theta^{2}, \\
\gamma_{1}(\theta)=13.618-0.631 \theta+0.007665 \theta^{2}, \\
\gamma_{2}(\theta)=5.896-0.258 \theta+0.00355 \theta^{2}
\end{gathered}
$$


where $\theta$ is the incidence angle in degrees.

It is noted that under development of the model, we have corrected the initial up-wind NRCS curve from [5] at the incidence angle of $30^{\circ}$ to eliminate exceeding of the down-wind NRCS value over the up-wind NRCS value at lower wind speeds and tending of the down-wind NRCS value to the crosswind NRCS value at higher wind speeds. The correction is based on the windwave tank study of Ka-band NRCS at the incidence angle of $30^{\circ}$ reported in $[6]$.

The FM-CW demonstrator system has an inclined one-beam fixed-position antenna, and so a circular flight should be completed to perform wind vector measurements. As the radar system is installed in a motorglider pod attached under the right wing, and the antenna beam axis is directed to the right side at an incidence (mounting) angle $\theta_{a . m}$ of $65^{\circ}$ (at a straight flight), the circular flight with the right roll should be completed (Fig. 2).

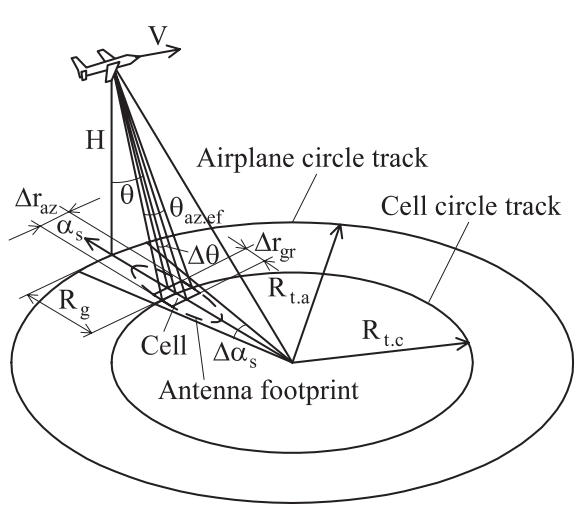

a)

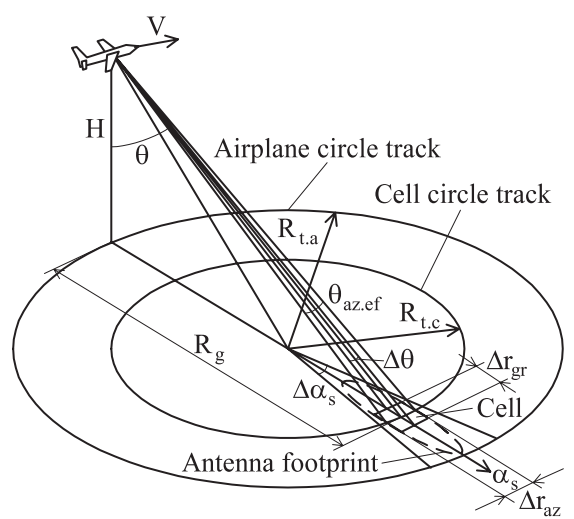

b)

Fig. 2. The circle flight geometry for measurement of the sea surface wind speed and direction: a) in the case of $R_{g}<R_{t . a}$; b) in the case of $R_{g} \geq R t . a$.

Two cases of the circular flight geometry for measurement of the sea surface wind speed and direction may occur. Fig. 2a shows the case of $R_{g}<$ $R_{t . a}$, and Fig. $2 \mathrm{~b}$ represents another case of $R_{g} \geq R_{t . a} ; R_{g}$ is the ground range, $R_{t . a}$ is the radius of the airplane turn, $R_{t . c}$ is the radius of turn of the selected cell middle point, $H$ is the platform altitude, $V$ is the platform velocity, $\Delta r_{a z}$ is the azimuth resolution, $\Delta r_{g r}$ is the ground-range resolution, $\theta_{a z . e f}$ is the effective (two-way) azimuth beamwidth, $\Delta \theta$ is the angular (twoway) resolution in the vertical plane.

Usually, the 360-degree azimuth space is divided into 72 or 36 sectors under the circle NRCS measurement. The azimuth size of a sector $\Delta \alpha_{s}$ is 5 or 10 degrees, respectively. The middle azimuth of a sector $\alpha_{s}$ corresponds to the azimuth location of the sector observed. The NRCS samples obtained from the sector and averaged over all measurement values in that sector give the NRCS value $\sigma^{\circ}\left(U, \theta, \psi_{s}\right)$ corresponding to the azimuth angle of the sector 
$\psi_{s}$ that is

$$
\psi_{s}=\left\{\begin{array}{lll}
\alpha_{s}-180^{\circ} \pm 180^{\circ} & \text { for } & R_{g}<R_{t . a} \\
\alpha_{s} \pm 180^{\circ} & \text { for } \quad R_{g} \geq R_{t . a} .
\end{array}\right.
$$

The wind speed can be found from the following equation

$$
U=\left(\sum_{i=1}^{360^{\circ} / \Delta \alpha_{s}} \sigma^{\circ}\left(U, \theta, \psi_{s . i}\right) /\left(\frac{360^{\circ}}{\Delta \alpha_{s}} a_{0}(\theta)\right)\right)^{1 / \gamma_{0}(\theta)} .
$$

To estimate wind direction, the main maximum value of the azimuth NRCS set

$$
\sigma_{\max }^{\circ}=\underset{i=1,360^{\circ} / \Delta \alpha_{s}}{\longrightarrow}\left\{\sigma^{\circ}\left(U, \theta, \psi_{s . i}\right)\right\}
$$

and the azimuth of the NRCS $\psi_{\sigma_{\max }^{\circ}}$ corresponding to it should be found. Then, the wind direction $\psi_{w}$ can be found

$$
\psi_{w}=\psi_{\sigma_{\max }^{\circ}} \pm 180^{\circ} .
$$

Also, the least squares method can be used to obtain a more precise location of the NRCS main maximum value, when a larger NRCS set is used. The function (1) at the measured wind speed value obtained from (4) is regarded to be the best approximation of sampled NRCS values within $\pm 60^{\circ}$ relative to its main maximum in azimuth, and then, the least value of summation results by the least squares method $S_{\min }$ can be written as follows

$$
S_{\text {min }}=\underset{j=0,360^{\circ} / \Delta \alpha_{s}}{\longrightarrow}\left\{\sum_{i=j-60^{\circ} / \Delta \alpha_{s}}^{j+60^{\circ} / \Delta \alpha_{s}}\left(\sigma^{\circ}\left(U, \theta, \psi_{s . j}\right)-\sigma^{\circ}\left(U, \theta, \psi_{i}\right)\right)^{2}\right\} .
$$

The azimuth of the NRCS $\psi_{s . j}$ corresponding to the least value of summation results $S_{\min }$ will be $\psi_{\sigma_{\max }^{\circ}}$, and the wind direction can be found from (6).

To investigate the performance of the proposed measuring algorithm, a simulation of the wind vector retrieval for the FM-CW demonstrator system in the scatterometer mode has been performed. The proposed algorithm has been tested at the wind speeds of 3 to $20 \mathrm{~m} / \mathrm{s}$ under averaging of a various number of NRCS samples.

\section{Conclusion}

The completed evaluation of the FM-CW demonstrator system has shown that the system can be applied for remote measurement of the sea surface wind vector in the scatterometer mode using the proposed algorithm.

The developed Ka-band backscatter model and the proposed algorithm can be used to produce an airborne radar system for operational measurement of the sea surface roughness characteristics and for safe landing of an amphibious aircraft on water, for example for search and rescue missions in coastal areas. 


\section{Acknowledgments}

We would like to express our sincere thanks to Prof. Dr. L.P. Ligthart, the International Research Centre for Telecommunications-transmission and Radar (IRCTR) at the Delft University of Technology for the research opportunity provided and to the Netherlands Organization for Scientific Research (NWO) for supporting Alexey Nekrasov with a research fellowship. 